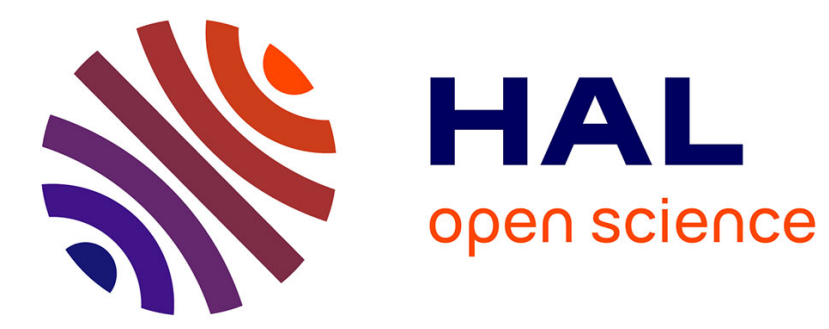

\title{
An Economic Approach To The Self: The Dual Agent
} Aileen Lotz

\section{To cite this version:}

Aïleen Lotz. An Economic Approach To The Self: The Dual Agent. 2019. hal-02314663

\section{HAL Id: hal-02314663 \\ https://hal.science/hal-02314663}

Preprint submitted on 13 Oct 2019

HAL is a multi-disciplinary open access archive for the deposit and dissemination of scientific research documents, whether they are published or not. The documents may come from teaching and research institutions in France or abroad, or from public or private research centers.
L'archive ouverte pluridisciplinaire HAL, est destinée au dépôt et à la diffusion de documents scientifiques de niveau recherche, publiés ou non, émanant des établissements d'enseignement et de recherche français ou étrangers, des laboratoires publics ou privés. 


\title{
An Economic Approach To The Self : The Dual Agent
}

\author{
Aïleen Lotz* \\ First Version: February 2011 \\ Current Version: October 2019
}

\begin{abstract}
This paper extends the notion of the rational agent in economics by acknowledging the role of the unconscious in the agent's decision-making process. It argues that the unconscious can be modelled by a rational agent with his own objective function and set of information. The combination of both the conscious and unconscious agents is called the dual agent. This dual agent presents rationally biased behaviors that may persist through aggregation and could be potentially measured. It also provides a theoretical approach to the emotionally-driven actions.

Key words: dual agent; conscious and unconscious, rationality; multi-rationality; emotions; choices and preferences; multi-agent model; consistency;

JEL Classification: B41, D01, D81, D82.
\end{abstract}

\section{Introduction}

The rational agent hypothesis is at the heart of economic theory. Developed among the neo-classical school during the middle of the nineteenth century, this hypothesis states that economic agents are rational, meaning that they choose their actions in order to maximize their utility. By systematically disregarding all nonrational behaviors, the theory has allowed economics to abstract itself from the vagaries of human nature and, as such, has been a powerful tool in the reshaping of the discipline as a natural science. While other social scientists were adamant in their quest to model human nature, economists created a world in which agents are perfectly rational, always optimize to the best of their knowledge and make no systematic errors.

Critics of this perfect rational man have been lurking in the economic literature for more than fifty years. They rightly point out that models based on such assumptions have blatantly and repeatedly failed to foresee any market crash or other economic disruption from the theoretical equilibrium. They stress the fact that economics should acknowledge the findings of other social sciences and build on them, rather than going on assuming improbable hypothesis.

This stream of criticisms has materialized in the behavioral branches of both Economics and Finance, which seek to suggest mathematical alternatives with firm psychological foundations to rational assumptions. However, it ignores the fact that psychology suffers from its own evils. The empirical approach adopted by the discipline condemns its theories to be regularly rejected or amended according to new empirical findings. Models based on fully rational microeconomic behavior usually yield results that are richer, deeper and more interesting than those achieved by behavioral models. This is probably why despite its grossly caricatural assumptions, the rational agent is still so much in favor in economics.

To put things differently, Economics may have been wrong in assuming one monolithic conscious agent. It has gone one step too far in its quest of simplification by assuming the self as a single rational agent. It does not follow, however, that its global approach is wrong.

In this paper, we step back and show that the inability of the rational agent hypothesis to account for irrational behaviors and psychological biases can be corrected once an additional unconscious agent is

*Ä̈leen Lotz: Cerca Trova, BP 114, 38001 Grenoble Cedex 1, France. E-mail: a.lotz@cercatrova.eu 
added along a conscious agent within each individual. The dual agent approach drops the apparently logical assumption of a single agent governed by a single conscious (see Becker 1962, 1968 for instance), and opts for two fully rational and coexisting agents. In order to do so, it seeks to determine the theoretical conditions under which these agents could be rational, act simultaneously using the same utility, and end up having a different action.

So that, whereas Behavioral approaches use psychology to inform economics and amend theoretical economic rationality according to empirical findings (Balthussen 2009; Thaler 1993; Mullainathan and Thaler 2001; and Camerer et al. 1999, 2003, 2004), the dual agent approach allows economics to inform psychology.

Of course, we are well aware that economic models of inconsistent behaviors have long considered the agent as a set of interacting sub-agents (see Ross 2010). These models use dual or multiple selves acting synchronically and/or diachronically to achieve different objectives, such as Akerlof's case of procrastination (1991, 2002). Usually, two selves are distinguished. A long-run self imposes costs on a short-run self and controls or manipulates his behavior (see Fudenberg and Levine 2006). The agent is time inconsistent since the short-run self departs from the plan designed by the long-run self.

This is particularly true in the Pico-economic framework (see Ainslie 1991, 1992; Ross 2010 and references therein for an account) that considers a sort of random successive self, depending on the state of nature assumed. Some further distinctions between selves can be introduced: one may control or correct some impulsive decisions of his predecessors. Thus, the role of the unconscious processes appear as a mere unknown mechanism modifying the short-term utility, implying time inconsistency biases in behavior and apparent successive selves.

The notion of dual agent proposed in this paper differs from the above since our goal is precisely to model these very unconscious processes. Only by describing some rational unconscious behavior can we hope to achieve this. We consider that the agents' patterns of behavior are permanent and active at all stages of the decision's and action's processes. In such a setting, unconscious processes are neither modelled as biases within the agent's mind nor by stochastic modification such as state of nature, but naturally emerge due to the coexistence, alongside the conscious, of a fully rational agent interpreting the external signals according to his own grid of lecture. There are no such thing as successive selves, but rather two (or several) interacting agents. Time inconsistency and biases only emerge as a result of these interactions. The unconscious agent we consider here is an agent that is absent and ignored from the standard models, and which specifically induces, through his action, the changes in behavior and the apparently successive selves.

Closer to our approach is Simon $(1982,1986)$. He stresses the importance of utilities, beliefs and expectations in economic rationality, which require an empirically founded theory of choice, that in turn needs to specify what information decision makers use, and how they actually process it. This has been the route taken here. Considering the unconscious as a rational agent permanently acting alongside the conscious in a simple model required to specify his information. The economical approach presented here establishes a minimal framework for the description of the unconscious as a rational agent.

The paper is structured as follows. The first section presents and discuss the dual agent hypotheses. Section 2 develops the model. It specifies the context, the objectives of both the conscious and unconscious agents and the resulting action of the combined dual agent. Section 3 presents the results of the model. Section 4 puts the results in perspective. Section 5 concludes.

\section{The Dual Agent Hypotheses}

This paper will describe two agents, the conscious and the unconscious, and their possible interactions within a single economic agent, the dual agent. In this section, we present the general specificities of each of these agents.

\subsection{Conscious and Unconscious agents}

The conscious agent considered here is the neo-classical rational agent. He apprehends the parameters of the reality through noisy signals and chooses his actions to optimize his welfare given all the information at his disposal. Since this conscious agent will turn out to be the quite familiar, we will rather dwell on the modeling of the unconscious agent. 
In its very general meaning, two type of actions can be qualified as being unconscious.

First, the actions that are not originating from the conscious mind, but that punctually emerge to the conscious mind, such as dreams, slips of the tongue, etc (see Freud 1899). These actions are conscious, but are said to be unconscious in that they reflect the activity of a part of our self that we do not control, whose actions we do not or cannot control or endorse, but that seems to reveal alternate logic, tastes or priorities.

A second, more straightforward acceptation of the term unconscious actions are those actions that are literally un-conscious, or non-conscious. They refer to the activity that is decidedly beyond the grasp of the conscious mind (for instance see Naccache 2009 or Armony and Vuilleumier 2013). This could refer to a purely physiological or neurological unconscious, so to speak, the unconscious that purely governs our mental and/or physiological processes, such as breathing, hunger, instincts, reflexes, etc.

If we were to focus on the first definition of the unconscious, and exclusively consider the part of the mind that is not conscious, but can become conscious at some point, we could reduce the economic agent to the traditional rational agent, whose perception of reality is only impaired by his imperfect information, or his own inability to counter some inappropriate behaviors. This is the path followed by Behavioral Economics. Boots of irrationality are seen as punctually superseding the rational agent's actions. The unconscious is taken into account, but not modeled as a rational and permanently acting agent in his own right, alongside the conscious.

Alternatively, if we reduced the unconscious to the second part of the former definition, i.e. if it were the part of psychic activity beyond the reach of the conscious mind, the parameters of the reality the unconscious agent could react to through his action would be radically beyond the knowledge of the conscious agent. Both the conscious and the unconscious agents could, and would indeed, coexist in radically independent worlds. Our purpose would be trivial, the action of the resulting - combined - economic agent being the sum of two distinct and independent actions performed by two distinct agents, endowed with radically different knowledge and clearly defined fields of competence. Besides, in such a context, the action of this unconscious would be of slight interest to the economist.

To us, the best approach is therefore to describe the unconscious as a second, permanent, fully rational agent, acting alongside the conscious, yet distinct from the latter. It is the approach we shall take in this paper. Whatever the actions these two agents, conscious or unconscious, can take separately, there is only one physical agent that will act. Therefore we cannot treat these two agents separately. We will call the combination of the conscious and the unconscious agents acting alongside and simultaneously the dual agent.

\subsection{Utilities}

Could the utilities of the conscious and the unconscious agents differ? The unconscious performs, among various other tasks, all the physiological functions that guarantee the individual's survival at their best routinely. This survival is guaranteed on the basis of a body of past knowledge, acquired at birth or through time by the individual, that may be modified by events, so that it may evolve through time. However, being a mix of genetic heritage, physiological regulatory processes, instincts, reflexes, social conditioning, among other relevant factors, it can be supposed to be either constant or strongly backward-looking.

Yet the term survival is very limited and should be replaced by the term wellbeing: the survival being a wellbeing a minima, it can be seen as the least desirable of all other preferable outcomes. Such a setup is, in itself, the guarantee that the individual survival will be, at worst, satisfied. So that we can safely state that the unconscious agent optimizes the wellbeing of the individual.

But experience informs us that this objective is a priori shared by the conscious agent. Naturally, this often turns out to be a more subjective wellbeing than an objective one. To preserve our health, we should definitely eat less, drink less, drive more carefully, or quit smoking. But our subjective wellbeing somehow imposes that we eat or drink as much as we like of what we prefer, drive too fast and go on smoking. Some may at times want to suppress themselves altogether, yet this could also be seen as a mere extreme version of a conscious quest of wellbeing.

We are driven to conclude that both the conscious and the unconscious agents have the same utility, the preservation of the individual wellbeing. And indeed, in this paper it is not the utility per se that distinguishes both agents, but the way they perceive their environment. This will become more evident by specifying further each agent's characteristics and their resulting grids of lectures. 


\subsection{Grids of lecture}

We have seen in the above that the conscious agent must have exactly the same utility as the unconscious agent. Yet economic theory predicts that when the same set of information is shared by two rational agents having the same utility, their actions should be identical.

In order to explain distinct behaviors between two agents, and for these actions to be the result of some kind of optimization, we must allow some difference of information to emerge between the conscious and unconscious agents. We assume that the difference in information between the conscious and unconscious agents does not arise from the parameters of the reality, but rather from the way they are processed by each agent, and that this directly derives from each agent's function.

We will suppose that the defining role of the unconscious is to scan the information - both internal and external - collected through the body perceptive modes. The unconscious may then recognize and respond to the parameters collected, before eventually transmitting all or part of this set of data to the conscious. This setup allows the unconscious to respond to a set of relevant characteristics before the conscious being even aware of a situation. It also allows for the conscious to be endowed with most, although maybe not all, of the parameters of the reality listed by the unconscious. This general setup is in line with the current acception of neuronal processes, that identify the brain as a machine set to represent attributes and mental objects. Our perceptive modes are a mere collection of mental processes seeking to extract the relevant information to the representation they are supposed to elaborate. neuronal networks, that share the same encoding information properties, then process this information. As such, they constitute representational systems of external and internal information. We also assume that the conscious is able to group and recognize these characteristics, name them, and organize them in multiple subsets forming one coherent set.

To illustrate this point, let us analyze two practical examples. Let us suppose you are standing in front of a building. You can see the building. That is to say that your conscious is able to identify a building. Yet the conscious has only been able to reach this conclusion thanks to the unconscious. Indeed, the unconscious has first collected a list of data through his perceptive channels. In front of the building, this list of data could be for instance: thing, static, big, rectangular, with dark holes, tiles, inhabitants, etc. Or let us suppose you are stuck in a traffic jam. Your car is at the stop, just behind a big truck. Once again, this is only what you can see, that is what your conscious as identified as a truck. To come to this conclusion, your conscious has processed a list of characteristics perceived by your unconscious, such as thing, in front of me, rectangular, with wheels, small mirrors, metal plate, number written at its back, smoke coming from a metal tube, etc.

From the above lists of characteristics, your conscious extracts the information that this thing in front of you is a building or a truck. From the context, he is able to deduce that these mirrors must be some rear-view mirrors, the metal plate a licence plate, the metal tube a muffler, and that this thing in front of you with wheels, rear-view mirrors, licence plate, and muffler, must be a truck. Following the same logic, he has deduced that this rectangular and static shape, with its roof, walls, windows and inhabitants must be a building.

\subsection{Implication}

The fact that the conscious treats some information collected by the unconscious has one important consequence. It implies that the unconscious must be an always operating-agent : his actions must be continuous. This in turn justifies the stance taken in this paper. The very fact that the conscious should be aware of the reality is the very proof that the unconscious is performing his function: listing the parameters of reality, analyzing and transmitting them to the conscious.

Besides, the fact that the unconscious and conscious agents share some or all signals, but interpret or weights them differently leads us to reinterpret what has been said on utilities. Even though conscious and unconscious have the same utility, the signals they use to optimize these utilities lead them to potentially radically different actions. Apparently, everything happens as if they had optimized different utilities.

However, these sole assumptions are not sufficient to induce any difference between the conscious and unconscious' actions. To induce a real difference between each agent's specific actions, we must suppose that they perceive the same reality in two distinct ways, and, consequently, treat the incoming information differently. In this paper, the critical difference between the conscious and unconscious lies in their respective perception of time. 
This distinction can be drawn from the very role of each agent. The unconscious scans and reacts to parameters of the reality, before eventually passing on some of this information to the conscious. He is also bound to maintain some specific equilibria within the body on a permanent basis. Each and every second, vital operations are performed, that cannot be postponed. Whereas the conscious can take time into account, and delay some or all of its actions, the unconscious is bound to act on a permanent, continuous basis so to speak. So that the laps of time on which each agent, conscious and unconscious, operate is radically different, and that we can safely assume that the unconscious short, medium and long term are infinitely shorter than the conscious ones.

The role of the unconscious is also to spot and react to potentially harmful situations. Some are predefined and passed from generation to generation through instincts, some are acquired through early and/or repeated experiences. In order to perform this function, the unconscious cannot discard any element of information. Whereas the conscious acts as a selecting device and tends to discard information once it has been processed, the unconscious never discards what he perceives, since it could always turn out to be relevant.

Two specific and very different agents emerge from these assumptions. In terms of information, the unconscious is clearly more informed than the conscious. He has access to a much wider and deeper perception of the parameters of the reality than the conscious. Yet it does not follow that the unconscious is better informed than the conscious. Indeed, the unconscious lacks the time dimension associated with the conscious activity. Naturally, strictly speaking, the unconscious takes time into account, but his time span is much shorter than the one of the conscious. His function is to preserve and come back to an equilibrium. Thus, by many aspects, the unconscious can be considered as a static agent. The conscious on the contrary can be considered as a dynamic agent. First of all, the conscious takes time into account, and his plans can be modified according to the continuously evolving parameters of the reality. Besides, the conscious can always postpone or modify his tasks depending on the environment.

This clearly suggests that, although the unconscious presented here can indeed be seen as a rational agent, the way it processes its information will trigger radically different actions from those of the conscious.

\subsection{Mutual awareness}

How do the conscious and unconscious perceive one another within the dual agent? In the present paper, we have supposed that the conscious and unconscious ignore each other's presence. This stance requires some explanations.

The conscious is, by definition, the least informed of the two agents. This directly results from the role of the unconscious, which scans the parameters of the reality before transmitting eventually some of them to the conscious. In this setup, the conscious is bound to ignore many of the parameters the unconscious is dealing with, and that are specific to his activity.

For example, the conscious will ignore most of the physiological processes the unconscious is performing constantly. Actually, there is no reason to assume that the conscious is aware of the fact that an other - and possibly more informed - agent exists along his side.

The unconscious is the most informed of the two agents in terms of scope of information. Yet it does not follow that he is fully and perfectly well informed. This results from the unconscious' grid of lecture. Because the unconscious ignores the time dimension of events, he has no reason to suspect that an alternate grid of lecture exists, nor that the conscious, using all information at his disposal, should know more or better than he does.

The overlapping nature of the unconscious and conscious knowledge - the set of parameters, actions, even utilities, could lead the unconscious to ignore the conscious' action, and vice-versa. Because the difference between the conscious and unconscious lies within their respective perception of time, they have no reason to suspect that the other agent perception could be more accurate than their own.

We have shown in the above that the unconscious has his own set of information, utility, and action, and that he can be considered as both economically rational and different from the conscious. We have furthermore mentioned that the conscious can be seen as the neo-classical rational agent. Let us now see how these two rational agents can be modeled as one single agent, the dual agent. 


\section{The Model}

In the following, we shall call conscious and unconscious the two rational economic agents, and the dual agent, the agent resulting from the combination of these two agents within a single individual. We will present the reality, a benchmark case, and the context faced by each agent, conscious and unconscious respectively, before specifying their information sets and utilities. We will then present the full model of the dual agent, along with its optimization. To simplify the matter, the dynamics of the model is left for further research.

\subsection{The reality}

Both conscious and unconscious actions suppose an external situation in which actions take place. We will call this environment the reality. It is described quantitatively by means of parameters. These parameters can refer to various elements such as social interactions, sets of behaviors, agents, costs, external conditions, interactions. All these constitute the environment the conscious, the unconscious and the dual agent face and are confronted to.

We will call $\theta$ this set of parameters describing the reality. The reality $\theta$ is a vector:

$$
\boldsymbol{\theta}=\left(\theta_{1}, \ldots \theta_{n}\right)
$$

Note that each parameter $\theta_{i}$ could be seen as a vector of characteristics that fully describes $\theta_{i}$. However, this refinement will be ignored here.

\subsection{Benchmark case: one fully informed agent}

To later discuss our results, we need to introduce a fictitious benchmark case that could be defined as the optimization of a fully informed agent. This agent would optimize his wellbeing depending on the true parameters of the reality:

$$
W\left(a, \theta_{1}, \ldots \theta_{n}\right)
$$

This would trigger an optimal action $a^{o p t}$. For the sake of simplicity, we will assume this action to be a linear combination of the parameters of the reality, so that $a^{o p t}$ will be a linear function of the parameters :

$$
a^{o p t}=\sum \alpha_{i} \theta_{i}
$$

The optimal action is the weighted sum of the parameters of the reality, where the $\alpha_{i}$ can be seen as transformers that translate the quantitative parameters of the reality into an action.

\subsection{The conscious}

The utility and optimal action of the conscious agent is similar to our benchmark case. However the conscious agent is not fully informed and has a specific grid of lecture. We assume that he can at best grasp part a set $\tilde{\boldsymbol{\theta}}$ defined as:

$$
\tilde{\boldsymbol{\theta}}=\left(\theta_{1}+\varepsilon_{1}, \ldots, \theta_{n}+\varepsilon_{n}\right)
$$

where the parameters $\varepsilon_{1}, \ldots, \varepsilon_{n}$ encompass all types of mistakes made by the conscious: mistakes in perceptions, lack of information for instance. As a consequence, if alone, he would process the parameters of reality and optimize his expected wellbeing to the best of his knowledge :

$$
E^{c} W\left(a, \theta_{1}, \ldots \theta_{n}\right)
$$

Where $E^{c}$ denotes the expectations given $\tilde{\boldsymbol{\theta}}$. Thus, the conscious will depart from the benchmark optimal action and set his action to:

$$
\begin{aligned}
a & =E^{c} a^{o p t}=\sum \alpha_{i} E^{c} \theta_{i} \\
& =\sum \alpha_{i}\left(\theta_{i}+\varepsilon_{1}\right) \\
& =a^{o p t}+\varepsilon
\end{aligned}
$$


where $\varepsilon$ is the overall mistake induced by the conscious' incomplete information:

$$
\varepsilon=\sum \alpha_{i} \varepsilon_{1}
$$

Action $a$ is in fact the standard optimization of the rational agent, where imperfect information can lead the agent to take erroneous decisions.

\subsection{The unconscious}

Recall that, in the present setup, the unconscious' distinctive role is to list characteristics perceived in the world that surrounds each individual. He performs this action by collecting the information of the body's various perceptive modes. So that the unconscious does not perceive the world as the conscious does, but first and foremost, as a pure list of parameters.

Besides, because he is a static agent, the unconscious, although rational, significantly departs from the conscious in his perception of his wellbeing. He does not consider situations as they occur to the conscious, but as a set of small units of meaningfull elements, that he recombines and interprets freely, to compare them to a specific set of predefined and potentially harmfull situations.

The unconscious must therefore be modeled as an agent reacting to a re-created and distinct situation from the actual one, so that the situation the unconscious is facing can be characterized by its own subset of parameters $\theta^{u}$, defined as:

$$
\boldsymbol{\theta}^{u}=\left(\theta_{1}^{u}, \ldots \theta_{n}^{u}\right)
$$

These parameters are the set of elements recognized as meaningful by the unconscious while analyzing the reality.

The unconscious systematically and continuously analyzes the present context and confronts it to his own parameters. In turn, the recognition of part or all of these parameters within the present context will trigger the unconscious' reaction.

However, because the unconscious decomposes the reality in small units and interpret them according to his own grid of lecture, some of these parameters may be overlooked by or simply unknown to the conscious, so that $\boldsymbol{\theta}^{u} \neq \widetilde{\boldsymbol{\theta}} \neq \boldsymbol{\theta}$.

Conscious and unconscious are therefore endowed with dissymmetric sets of information. The conscious perceives the reality through the fog induced by his incomplete information, while the unconscious keeps on analyzing the reality according to a mainly backward-looking grid of lecture.

Once the $\theta^{u}$ are recognized, the unconscious will seek to maximize a wellbeing $W^{u}=E^{u} W$, that can be written:

$$
E^{u} W=W^{u}\left(a, \theta_{1}^{u}, \ldots \theta_{n}^{u}\right)
$$

This wellbeing depends on the perceived situation. Here again, because the unconscious is backward-looking and mostly reacts to past situations. The wellbeing $W^{u}$ can be seen as an obsolete wellbeing, inducing actions that actually optimize real but past situations. His actions are therefore bounded to be suboptimal in the present context. We also assume that $W^{u}$ is quadratic in the $\left(\theta_{1}^{u}, \ldots \theta_{n}^{u}\right)$. His optimal action would be $E^{u} a^{o p t}=\sum \gamma_{i} \theta_{i}^{u}$, i.e. a combination of the perceived parameters by the unconscious.

\subsection{The dual agent}

The combination of the conscious and the unconscious is the dual agent.

Being a combination of two agents, this dual agent reacts to two sets of parameters : the parameters within the reality that are processed by the unconscious, on the one hand, and those significant to and processed by the conscious, on the other hand.

Therefore the dual agent will, as a single agent, optimize a combination of wellbeing: $W$ and $W^{u}$, the wellbeing of the conscious and the unconscious respectively:

$$
(1-\beta) E^{c} W\left(a, \theta_{1}, \ldots \theta_{n}\right)+\beta E^{u} W\left(a, \theta_{1}^{u}, \ldots \theta_{n}^{u}\right)
$$


where $\beta$ is the weight of the unconscious in the process of choice.

The parameter $\beta$ describes the "power of intrusion" of the unconscious in the dual agent's utility. When $\beta=0$, the action boils down to the standard benchmark case, in which the dual agent is the rational, conscious, agent. When $\beta \neq 0$, the higher the $\beta$, the more permeable the dual agent action is to the unconscious analysis of the reality, or alternatively, the more does the reality fit the unconscious grid of lecture. $\beta$ is therefore dependent on the parameters $\theta$ and $\theta^{u}$, and in turn, on the personal history of the dual agent. As such, it can evolve over time.

Stating a single, combined, utility for two agents may seem awkward. Game Theory would model these two agents as each having his own action and playing one against the other. However, in this model, Game Theory does not apply: only one agent, the dual agent, is playing. Yet this single action must nonetheless be the result of a combination of two utilities, and two individual independent actions. Indeed it is our assumption in this paper that they ignore one another. Moreover, given their respective perception of time, these two agents have no reason to suspect that their action should be different. The dual agent's action is therefore the result of the combined actions of the conscious and the unconscious, and this result cannot at least in the context of this paper - be reduced through Game Theory.

Formally, the optimal action taken by the dual agent is a combination, at least in first approximation:

$$
\begin{aligned}
a & =(1-\beta) E^{c} a^{o p t}+\beta E^{u} a^{o p t} \\
& =(1-\beta)\left(a^{o p t}+\varepsilon\right)+\left(\beta a^{o p t}+\beta\left(\sum \gamma_{i} \theta_{i}^{u}-a^{o p t}\right)\right) \\
& =a^{o p t}+(1-\beta) \varepsilon+\beta \sum\left(\gamma_{i} \theta_{i}^{u}-\alpha_{i} \theta_{i}\right)
\end{aligned}
$$

We will interpret this equation in detail in the result section.

To conclude this section, let us note first that, in the above, we have totally distinguished the conscious and unconscious perceptions of reality. It would actually be more accurate to consider the unconscious has been able to blur the perception of the conscious. This corresponds to replacing the conscious' perception of the parameters:

$$
\tilde{\boldsymbol{\theta}}=\left(\theta_{1}+\varepsilon_{1}, \ldots \theta_{n}+\varepsilon_{n}\right)
$$

by a blurred combination

$$
\tilde{\boldsymbol{\theta}}^{\prime}=\left(\theta_{1}+\lambda_{1} \theta_{1}^{u}+\varepsilon_{1}, \ldots \theta_{n}+\lambda_{n} \theta_{n}^{u}+\varepsilon_{n}\right)
$$

In this context, an analysis would lead to a net gain of information for the conscious, by re-establishing the true signals $\tilde{\boldsymbol{\theta}}$.

Note also that the timing of the action could be refined. Each agent could act separately or sequentially, and in turn observe the result of these two actions. Alternately, the unconscious could blur the parameters of the conscious with his own parameters, thereby modifying the conscious' wellbeing. Yet these situations would be equivalent, and are indeed more conveniently modeled through a single, dual agent's wellbeing, that combines both the conscious and unconscious agents' wellbeing.

\section{Results}

Equation (6) shows that the action of the dual agent can be broadly defined as the sum of two terms : an optimal social action, and a bias to this optimal social action.

The first term, $a^{o p t}$, is the optimal action of the dual agent. It encompasses the actions of the conscious and the unconscious that would be adequate when facing a given environment. It can be defined as the optimal social choice.

The two other terms $(1-\beta) \varepsilon$ and $\beta \sum\left(\gamma_{i} \theta_{i}^{u}-\alpha_{i} \theta_{i}\right)$ blur this optimal social action:

The second term of equation $(6),(1-\beta) \varepsilon$, is the usual white noise of standard economic models. It is unavoidable and results from the usual conscious' lack of information about the parameters of the reality. It represents the overall noise that impairs the conscious' choice, and produces a sub-optimal and totally random outcome.

The second term of the bias, $\beta \sum\left(\gamma_{i} \theta_{i}^{u}-\alpha_{i} \theta_{i}\right)$, represents a pure action of the unconscious. Unlike the first term of the bias, it is not random, and represents a systematic bias with respect to $a^{o p t}$. 
This term should not be sub-optimal since the unconscious optimizes a wellbeing. Yet the wellbeing of the unconscious, $W^{u}$, does not merely optimize the real, actual situation faced by the agent, but rather a set of past atemporal situations perceived in the present situation. It only seldom represents an optimal action vis-à-vis the present situation, as seen by the conscious, but should rather be seen as an effective loss for the conscious. Its impact on the dual agent's action will depend on the weight given to $W^{u}$ within the dual agent's optimization.

To the dual agent however, this bias is part of his optimization, and cannot be seen as a loss. It is nonetheless inefficient on a social point of view, since it is biased toward the unconscious' optimum.

What is this bias composed of? Note that it is a combination of the differences between the parameters perceived by the conscious and those perceived by the unconscious. It results from the difference of grid of lecture between conscious and unconscious, and reflects the discrepancies that arise between mere facts and the way they are interpreted by the conscious and the unconscious respectively.

While the parameters of the conscious are real and observable, the parameters of the unconscious are, at least partly, discarded or unobservable to the conscious, since by definition the $\theta_{i}^{u}$ are projections of the unconscious on the parameters $\theta_{i}$ in an atemporal space of parameters. $\theta_{i}^{u}$ is therefore the result of an interpretation performed by the unconscious and, as such, incomprehensible to the conscious. This overall bias can therefore be defined as an action performed by the dual agent, directly observable to the conscious. It is a departure from the optimal action, in that it would not necessarily be required from the situation as such. The conscious does not control it, since it is induced by an unconscious activity, and is the result from the misinterpretation of a present situation by the unconscious.

Let us underscore that if this bias can be a material action, it is not necessarily bound to be so. It could very well be an immaterial action. It could be a slip of the tongue, for example, but it could also be a thought, an emotion, a feeling, an exclamation, etc. Although it may not be fully adequate, let us for now call it the emotive part of the agent's action.

Last but not least, this bias in the action of the dual agent will not necessarily disappear when aggregating over agents. It will depend on the nature of the bias. Actually, if its trigger is a common scheme among individual, and not a personal feature, it will sum up over a population. This can give some rationale to some common seemingly irrational behaviors.

\section{Discussion}

This paper innovates in showing that the action of two purely rational agents optimizing according their own grid of lecture within a single dual agent results in a perfectly optimal action, an error term, and a bias to this optimal action.

The optimal action $a^{o p t}$ is a combination of the actions of the conscious and the unconscious that would adequately respond to reality. It can be seen as the action of a perfectly informed dual agent. This action is subject to errors, notably due to the dual agent's lack of information. The last part of the action is a bias to the optimal action. It is not irrational per se, however it is not factually optimal since it is not relevant to the present situation, and as such it can seem irrational.

What is the nature of this bias? Recall that the bias has been defined as an action performed by the dual agent, directly observable to the conscious but not controlled by him. We have called this bias the emotive part of the action.

Note that our model does not distinguish between behaviors and emotions. Both could be part of the optimal action. When facing a danger, it could be optimal for any individual to be scared, i.e. to experience an emotion, and run, i.e. to adopt a certain behavior. And alternately some actions taken under emotional stress can be seen as biased and irrational. So that both behaviors and emotions can be part of the bias to the optimal action, rather than part of the optimal action itself. Ultimately, thoughts too are highly dependent on the unconscious, and few are absolutely devoid of emotive content. So that emotions, behaviors and thoughts could all be part of the bias, and as such be explained by the introduction of a second agent, the unconscious, endowed with an alternate and specific grid of lecture of the reality.

By encompassing emotions, cognitions and behaviors in the bias, our model bridges the gap between emotion theorists and economists (see Elster 1998), and provides common grounds to psychology and economics, which is supported by a constant litterature (Smith 1859; Tarde 1902; Katona 1951; Rabin 1998, 
2005). An implication of our views is the following: since the bias encapsulates the difference of information between conscious and unconscious, the model suggests that emotions will be regulated once the information gap is filled, i.e. when the information of the unconscious will be shared by the conscious.

To illustrate this point, let us consider the example of anger. How does anger evolve? At first, it is manageable. However, if the source of our anger does not disappear, we will eventually utter it out. And at some point, our anger will suddenly disappear. The present model suggests that anger, as any emotion, is a bias to an optimal action. It is caused by an alternate perception of the unconscious and is encapsulated in the bias to the optimal action. When uttered out, the unconscious information is explicitly expressed and shared or revealed to the conscious. The information gap is filled and anger disappears.

Let us stress that the hypothesis of an imperfectly informed conscious does not a priori allow him to suspect the presence of a bias in his optimization. Without prior knowledge of the unconscious, the conscious would deduce ex post - after revelation of the true parameters - that a suboptimal action resulted from his misestimation of the situation measured by $(1-\beta) \varepsilon$. Repeated mistakes could lead him to the conclusion of a lack of estimation power.

However, the bias could be suspected and quantitatively estimated. The presence of the systematic bias $\beta \sum\left(\gamma_{i} \theta_{i}^{u}-\alpha_{i} \theta_{i}\right)$ could be measured by the correlation of the mistakes in a series of similar situations. Indeed, measuring the average of his actions through an extended period of time and over a large sample of similar actions could lead to eliminate random errors and measure an average action

$$
\bar{a}=a^{o p t}+\beta \sum\left(\gamma_{i} \theta_{i}^{u}-\alpha_{i} \theta_{i}\right)
$$

Ex-post, when $a^{\text {opt }}$ is revealed, the systematic bias could be measured. We can even venture that the $\theta_{i}^{u}$ could be partly retrieved. By examining the biases over a sufficiently large sample of events, one could retrieve, a minima, some structural similarities among various situations, and postulate a structural form for a set of $\theta_{i}^{u}$. This would help determine the form of the triggers inducing the unconscious reaction. However, this would require to abstract oneself from the contingencies of the present situation and seek similarities within situations.

To conclude this discussion, let us point out that the notion of dual agent presented here has been at the root of several works developing models of interactions both inside a single economic agent, and between several agents. These subsequent papers demonstrate the advantages there are in considering rational substructures in economic theory to describe apparent irrational behaviors, and validate the path taken here. Gosselin and Lotz (2012) and Gosselin et al. (2013, 2015) generalized the description of an economic agent as a set of several substructures with different grids of lectures, interacting permanently and possibly strategically (a related approach has been developed in Cunningham 2013). Depending on the strength of interactions stable equilibria or recurrent cycles of behavior appear, along with systematic apparent biases in behavior. These first applications further led the authors to develop a general theoretical framework describing dynamic interactions of a large number of economic agents (Gosselin et al 2017, 2018, 2019). The appearance of non-trivial phases and the individual dynamics of the agents in these phases can be derived for such systems. In particular, Gosselin et al (2017) applied this framework to model multiple selves and derived the various states of a multiple-self agent, i.e. the possible backgrounds of behavior in which the actions of an aggregated agent take place.

\section{Conclusion}

Modeling the unconscious as a second agent acting alongside the neo-classical rational conscious agent has proved to induce a bias in this dual agent's optimization. This bias, although rational and optimal under the dual agent's perspective, is actually sub-optimal with respect to the actual context. Insofar as it is generated by predetermined collective schemes, it could persist despite aggregation.

The dual agent framework represents an alternative to the economic agent, and allows to consider its actions under a psychological angle. It provides a useful theoretical framework to analyzing emotionally-

driven outcomes. Alternatively, it allows to consider psychological processes under an economical point of view.

The concepts that have been discussed here allow for a certain amount of extensions, both on theoretical and empirical grounds. On the theoretical side, the most obvious and immediate extensions are the intro- 
duction of dynamics, and interaction between conscious and unconscious, respectively. On the empirical side, tools to measure the bias in the dual agent's action should be developed. 


\section{References}

[1] Ainslie G (1991) Breakdown of Will. Cambridge University Press, Cambridge.

[2] Ainslie G (1992) Picoeconomics. Cambridge University Press, Cambridge.

[3] Akerlof G (1991) "Procrastination and obedience". American Economic Review 81:1-19.

[4] Akerlof G (2002) Behavioral macroeconomics and macroeconomic behavior. American Economic Review 92:411-33.

[5] Armony J, Vuilleumier P (2013) The Cambridge Handbook of Human Affective Neuroscience. Cambridge University Press, Cambridge.

[6] Baltussen G (2009) Behavioral Finance: An Introduction. Available at SSRN: https://ssrn.com/abstract $=1488110$ or http://dx.doi.org/10.2139/ssrn.1488110

[7] Becker G (1962) Irrational behavior and economic theory, Journal of Political Economy 70:1-13.

[8] Becker G (1968) Crime and Punishment: An Economic Approach. The Journal of Political Economy $76: 169-217$.

[9] Berg N, Gigerenzer G (2010) "As-if behavioral economics: Neoclassical economics in disguise?," MPRA Paper 26586, University Library of Munich, Germany.

[10] Camerer CF (1999) "Behavioral Economics: Reunifying Psychology and Economics." Proceedings of the National Academy of Sciences, 96:10575-77.

[11] Camerer C, Loewenstein G, Rabin M (2003) Advances in Behavioral Economics. Princeton University Press, Princeton.

[12] Camerer C, Loewenstein G, (2004) "Behavioral Economics: Past, Present, Future. Advances in Behavioral Economics". C. F. Camerer, G. Loewenstein and M. Rabin. Princeton University Press, Princeton: $3-52$.

[13] Camerer C et al. (2005) "Neuroeconomics: How Neuroscience Can Inform Economics". Journal of Economic Literature 43: 9-64.

[14] Cunningham T (2013) "Biases and Implicit Knowledge" MPRA Paper 50292, University Library of Munich, Germany. https://mpra.ub.uni-muenchen.de/50292/1/MPRA_paper_50292.pdf

[15] Elster J (1998) "Emotions and Economic Theory," Journal of Economic Literature, American Economic Association, vol. 36(1), pages 47-74, March.

[16] Freud S (1899/1900) Die Traumdeutung. Franz Deuticke, Vienna.

[17] Fudenberg D, Levine DK (2006) "A dual self model of impulse control". The American Economic Review, pp.96:1449-1476.

[18] Gilbert D, Fiske S, Lindzey G (1998) Handbook of Social Psychology, 4th edn. McGraw-Hill.

[19] Gosselin P, Lotz A (2012) "A dynamic model of interactions between conscious and unconscious," MPRA Paper 36697, University Library of Munich, Germany.

[20] Gosselin P, Lotz A, Wambst M (2013). "On Apparent Irrational Behaviors : Interacting Structures and the Mind," Working Papers hal-00851309, HAL.

[21] Gosselin P, Lotz A, Wambst M (2015). "From Rationality to Irrationality : Dynamic Interacting Structures," Working Papers hal-01122078, HAL.

[22] Gosselin P, Lotz A, Wambst M (2017) "A Path Integral Approach to Interacting Economic Systems with Multiple Heterogeneous Agents" Working Papers hal-01549586, HAL. 
[23] Gosselin P, Lotz A \& Wambst M (2018) "A Path Integral Approach to Business Cycle Models with Large Number of Agents" Working Papers hal-01893556, HAL.

[24] Gosselin, P., Lotz, A. \& Wambst (2019), "A Statistical Field Approach to Capital Accumulation" Working Papers arXiv:1909.11635.

[25] Hayek FA (1952) The Sensory Order. An inquiry into the foundations of theoretical psychology. University of Chicago Press, Chicago.

[26] Kahneman D, Tversky A (1979) Prospect theory: an analysis of decision under risk. Econometrica 47:263-91.

[27] Kahneman D, Knetsch JL, Thaler RH (1986) Fairness as a constraint on profit seeking. American Economics Review 76:728-41.

[28] Kahneman D (2011) Thinking, Fast and Slow. Farrar, Straus and Giroux, New York.

[29] Katona G (1951) Psychological Analysis of Economic Behavior. McGraw-Hill, New York.

[30] Mullainathan S, Thaler RH (2001) "Behavioral economics". In International Encyclopedia of SocialSciences, Pergamon Press, 1st edition, October 1, 2001: 1094-1100.

[31] Rabin M (1998) Psychology and economics. Journal of Economic Literature 36:11-46.

[32] Rabin M (2002) 'A perspective on psychology and economics', European Economic Review 46: 657-85.

[33] Ross D (2010) "Economic models of procrastination". In C. Andreou \& M. White, eds., The Thief of Time. Oxford University Press. pp. 28-50

[34] Simon H (1982) Models of Bounded Rationality. MIT Press, Princeton.

[35] Simon H (1986) "Rationality in Psychology and Economics," Journal of Business, University of Chicago Press, vol. 59(4), pages S209-24.

[36] Smith A (1759/2002) The Theory of Moral Sentiments, 6th edn, K. Haakonssen (ed.). Cambridge University Press, Cambridge.

[37] Tarde G (1902). La psychologie économique (2 vols). Alcan, Paris.

[38] Thaler R (ed.). (1993) Advances in Behavioral Finance. Russell Sage Foundation, NewYork.

[39] Kahneman D, Tversky A (1974) "Judgment under uncertainty : Heuristics and biases", Science 185 (4157), 1124-1131.

[40] Kahneman D, Tversky A (1981) "The Framing of decisions and the psychology of choice", Science 211 (4481), 453-458. 